\title{
Combustion simulations with accurate transport properties for reactive intermediates
}

\author{
Paul J. Dagdigian \\ Department of Chemistry, The Johns Hopkins University, Baltimore, Maryland 21218, \\ $U S A$
}

\begin{abstract}
The effect of employing accurate transport properties in simulations of freely propagating $\mathrm{H}_{2} / \mathrm{O}_{2} / \mathrm{He}$ and $\mathrm{CH}_{4} / \mathrm{O}_{2}$ flames is investigated. Transport properties for the collision pairs $\mathrm{H}_{2}-\mathrm{H}_{2}, \mathrm{H}-\mathrm{H}_{2}$, and $\mathrm{H}-\mathrm{H}$ are computed from published tables of collision integrals. Previous calculations in our group of transport properties for $\mathrm{H}-\mathrm{H}_{2} \mathrm{O}$ and $\mathrm{H}-\mathrm{O}_{2}$ have been extended to higher temperatures. In addition, new calculations on $\mathrm{H}-\mathrm{He}, \mathrm{O}-\mathrm{He}, \mathrm{H}_{2}-\mathrm{He}$, and $\mathrm{H}_{2} \mathrm{O}-\mathrm{He}$ are reported. Flame simulations with two sets of transport data were compared. The first set contained transport properties obtained with the conventional method of employing parameterized Lennard-Jones (12-6) potentials, while in the second set transport properties for the above collision pairs were substituted. A modest increase in laminar flame speeds, comparable to differences found when employing different chemistry models, was found for both flames.
\end{abstract}

Keywords: $\mathrm{H}_{2} / \mathrm{O}_{2} / \mathrm{He}$ and $\mathrm{CH}_{4} / \mathrm{O}_{2}$ flames; premixed flames; transport properties

\section{Introduction}

There has been great interest and activity on computationally simulating combustion in spatially inhomogeneous environments, such as internal combustion engines [1]. A significant effort has been invested by many groups on the development of chemical combustion models to describe reliably the temperature, and also pressure, dependence of the rate constants for relevant chemical reactions (see, for example, $[2,3,4,5,6]$ ).

The use of accurate transport properties is also essential in the reliable modeling of combustion in flames and other media. Sensitivity analyses by 
several groups suggest that the uncertainties in the transport properties can be as significant as the uncertainties in measured reaction rate constants $[7,8,9,10]$. Brown et al. [11] have extensively reviewed the methods that have been employed for the estimation of transport properties for combustion modeling.

The conventional approach for the estimation of transport properties has usually involved the use of isotropic Lennard-Jones (LJ) (12-6) potentials with the well and depth and length parameters $\epsilon$ and $\sigma$, respectively, determined through combination rules for the like pairs. In recent work, Jasper, Miller, and co-workers $[12,13]$ have fit LJ parameters to suitably spherically averaged ab initio potentials for alkane-helium and $\mathrm{H}, \mathrm{H}_{2}$, alkane- $\mathrm{N}_{2}$ interactions. As noted by Paul and Warnatz [14], the repulsive wall of a LJ (12-6) is generally steeper than the true potential, and this can lead to an incorrect computed temperature dependence of the transport properties.

Several groups $[15,16,17,18,19,20,21,22,23,24]$, including our own $[25,26,27,28]$, have computed transport properties for a number of collision pairs through quantum or classical scattering calculations employing ab initio potentials. Our particular interest has centered on the calculation of transport properties of collision pairs involving small transient species such as atoms and diatomic radicals for which accurate potential energy surfaces (PES's) are available or are calculated. We have found that the repulsive walls of PES's involving radical species are less steep than that of the LJ (12-6) potential. In some cases, for example $\mathrm{CH}_{2}-\mathrm{He}$ [26] and $\mathrm{H}-\mathrm{O}_{2}$ [26], the accurately computed transport properties are quite significantly different than those computed using assumed LJ (12-6) potentials, especially in their temperature dependence. These are systems for which the PES is strongly anisotropic or has a deep potential well.

It is worthwhile to go beyond sensitivity analyses of the importance of reliable transport properties and the comparison of accurate and LJ (12-6) computed transport properties. Dong et al. [8] have carried out combustion simulations of premixed $\mathrm{H}_{2}$ /air flames with updated diffusion coefficients to investigate their effect on computed extinction strain rates.

In the present study, we probe the effect of employing accurate transport properties in simulations of $\mathrm{H}_{2} / \mathrm{O}_{2} /$ He flames. Here, the chemistry is quite simple as there are only 9 chemical species that need to be included: $\mathrm{H}_{2}, \mathrm{O}_{2}$, $\mathrm{H}_{2} \mathrm{O}, \mathrm{H}, \mathrm{O}, \mathrm{OH}, \mathrm{HO}_{2}, \mathrm{H}_{2} \mathrm{O}_{2}$, and $\mathrm{He}$. With such a simple chemical system, it has been possible to include accurate transport properties for a significant fraction of the collision pairs, particularly those involving radical species. We 
have also investigated the effect of employing accurate transport properties on simulations of $\mathrm{CH}_{4} / \mathrm{O}_{2}$ flames, in which many more chemical species are present. Here, we compare laminar flame speeds in freely propagating flames, using different sets of transport properties. We find that the use of accurate transport properties has a noticeable effect on the computed flame properties.

This paper is organized as follows: The calculation of transport properties through quantum scattering calculations is briefly reviewed in the next section. The results of previous calculations are summarized, and transport properties for newly computed systems are presented. Section 3 describes our combustion simulations, and Sec. 4 presents computed flame speeds for freely propagating flames. The paper concludes with a discussion.

\section{Calculation of Transport Properties}

In our work $[25,26,27,28]$, we have computed transport properties through quantum scattering calculations. We present a brief review of the methodology employed.

In previous papers, we considered the full anisotropic atom-molecule PES's and computed collision integrals $\Omega^{(n, s)}$ for specific collision pairs, from which transport properties can be determined. The collision integrals are computed as Boltzmann averages of integrals of transport cross sections over the molecular rotational levels [27, 29, 30, 31, 32]:

$$
\Omega^{(n, s)}(T)=\frac{1}{q_{R}} \sum_{j_{i}}\left(2 j_{i}+1\right) \exp \left(-\varepsilon_{i} / k_{B} T\right) \Omega_{j_{i}}^{(n, s)}(T)
$$

where $j_{i}$ and $\varepsilon_{i}$ are the rotational angular momentum and energy, respectively, of the $i$ th molecular rotational level, $q_{R}$ is the rotational partition function, and $k_{B}$ is the Boltzmann constant.

The state-specific collision integrals $\Omega_{j_{i}}^{(n, s)}(T)$ in eq 1 are computed by integration over the collision energy $E$ of state-specific transport cross sections:

$$
\begin{aligned}
\Omega_{j_{i}}^{(n, s)}(T) & =\frac{1}{2}\left(\frac{k_{B} T}{2 \pi \mu}\right)^{1 / 2} \frac{1}{\left(k_{B} T\right)^{s+2}} \\
& \times \int_{0}^{\infty} E^{s+1} \exp \left(-E / k_{B} T\right) Q_{j_{i}}^{(n)}(E) d E
\end{aligned}
$$

where $\mu$ is the atom-molecule reduced mass. The state-specific transport

cross section $Q_{j_{i}}^{(n)}(E)$ in eq 2 is a sum of state-to-state cross transport sections 
over the final levels:

$$
Q_{j_{i}}^{(n)}(E)=\sum_{j_{f}} Q_{j_{i} \rightarrow j_{f}}^{(n)}(E)
$$

In eq 3, we have restricted the inelastic transitions to changes in the rotational level, and not vibrational transitions. Cross sections for the latter are much smaller than for rotational transitions.

The state-to-state transport cross sections in eq 3 are angle weighted averages of state-to-state differential cross sections:

$$
Q_{j_{i} \rightarrow j_{f}}^{(n)}(E)=\int\left(\frac{d \sigma}{d \Omega}\right)_{j_{i} \rightarrow j_{f}} \Phi_{n}(E) d \hat{R}
$$

where $\hat{R}$ is the orientation of the Jacobi vector $\mathbf{R}$. The weighting factors $\Phi_{n}(E)$ for $n=1$ and 2, as well as expressions for the state-to-state transport cross sections in terms of the $T$-matrix elements from time-independent quantum scattering calculations, have been given previously [26, 30].

It is customary to employ reduced collision integrals $\Omega^{(n, s) *}$ in the calculation of transport properties. These are related to the collision integrals given in eq 1 by $[29,11]$

$$
\sigma^{2} \Omega^{(n, s) *}\left(T^{*}\right)=\frac{F(n, s) \Omega^{(n, s)}(T)}{\left(k_{B} T / 2 \pi \mu\right)^{1 / 2}}
$$

where $T^{*}=k_{B} T / \epsilon$ and the factor $F(n, s)$ equals

$$
F(n, s)=\frac{4(n+1)}{\pi(s+1) !\left[2 n+1-(-1)^{n}\right]}
$$

The binary diffusion coefficient is related to the $(1,1)$ reduced collision integral through the relation

$$
D=\frac{3\left(2 \pi k_{B}^{3} T^{3} / \mu\right)^{1 / 2}}{16 N \sigma^{2} \Omega^{(n, s) *}}
$$

where $N$ is the number density of the gas. The following ratios of collision integrals are required for the calculation of thermal diffusion coefficients [33]:

$$
\begin{aligned}
& A^{*}=\Omega^{(2,2)} /\left(2 \Omega^{(1,1)}\right) \\
& B^{*}=\left(5 \Omega^{(1,2)}-\Omega^{(1,3)}\right) /\left(3 \Omega^{(1,1)}\right) \\
& C^{*}=\Omega^{(1,2)} /\left(3 \Omega^{(1,1)}\right)
\end{aligned}
$$


We have employed literature values, where available, for calculated collision integrals. Specifically, we have taken reduced collision integrals for $\mathrm{H}_{2}-$ $\mathrm{H}_{2}, \mathrm{H}-\mathrm{H}_{2}$, and $\mathrm{H}-\mathrm{H}$ interactions from the work of Stallcop and co-workers $[16,18]$. We have extended our published [25, 27, 28] collision integral calculations on the $\mathrm{OH}-\mathrm{He}, \mathrm{H}_{2} \mathrm{O}-\mathrm{H}$, and $\mathrm{H}-\mathrm{O}_{2}$ systems to higher collision energies so that collision integrals, including $\Omega^{(n, s)}$ for $(n, s)=(1,2)$ and $(1,3)$, can be computed to higher temperatures than $1500 \mathrm{~K}$, reported in these papers.

The subsections below describe new calculations we have carried out on several other collision pairs involving radical species. The collision integrals $\Omega^{(n, s)}$ for $(n, s)=(1,1),(1,2),(1,3)$, and $(2,2)$ for all the collision pairs considered are presented in the Supplementary Material (see Appendix A). We compare diffusion coefficients calculated with our collision integrals with those computed previously in section 2.5. Plots of the collision integral ratios $A^{*}$, $B^{*}$, and $C^{*}$ ratios are presented in the Supplementary Material.

\section{1. $H-H e$}

Middha et al. [22] have computed the temperature-dependent diffusion coefficient for the $\mathrm{H}-\mathrm{He}$ interaction through quantum scattering calculations employing an ab initio calculated potential energy curve. Since we would like to have collision integrals beyond just the $(1,1)$ term (see eq 7 ) needed for the diffusion coefficient, we have computed a new $\mathrm{H}-\mathrm{He}$ potential energy curve and calculated the collision integrals needed for the transport properties discussed above.

We employed the MOLPRO 2010.1 suite of computer codes [34] to compute the HHe potential energy curve by means of restricted coupled-cluster calculations with inclusion of single, double, and (perturbatively) triple excitations $[\operatorname{RCCSD}(\mathrm{T})][35,36]$. An atom-centered avqz basis set was used [37], with the addition of a set of mid-bond functions located midway between the atoms. A counterpoise correction was applied to correct for basis set superposition error [38].

\section{2. $\mathrm{O}\left({ }^{3} \mathrm{P}\right)-\mathrm{He}$}

We have employed the $\mathrm{OHe}^{3} \Sigma^{-}$and ${ }^{3} \Pi$ potential energy curves computed by Krems et al. [39] for the calculation of collision integrals for this system. The Hamiltonian for the interaction of an atom in $\mathrm{a}^{3} \mathrm{P}$ state with a closedshell atom was taken from Alexander et al. [40]. We have considered the individual spin-orbit levels of the $\mathrm{O}\left({ }^{3} P_{j}\right)$ collision partner and carried out the Boltzmann state average in eq 1 . The $\mathrm{O}\left({ }^{3} P\right)$ spin-orbit constant as 
assumed to be independent of the internuclear separation $R$ and taken to equal to the asymptotic atomic value.

\section{3. $\mathrm{H}_{2}-\mathrm{He}$}

Bakr et al. [41] have recently computed a highly accurate PES for the $\mathrm{H}_{2}-\mathrm{He}$ interaction. We have taken their fit of the 2-dimensional surface for which the $\mathrm{H}_{2}$ internuclear separation was fixed at the vibrationally averaged $\mathrm{H}-\mathrm{H}$ bond length, with the usual Legendre polynomial expansion describing the angular dependence of the PES [42]. This PES is not very anisotropic, and we used only the spherical average $(\lambda=0$ term) in our calculation of transport properties. In previous work [25, 27], we showed that taking the spherical average for $\mathrm{OH}-\mathrm{He}$ and $\mathrm{H}_{2} \mathrm{O}-\mathrm{H}$ yielded transport properties differing (by a few \%) from those computed with the full anisotropic PES.

\section{4. $\mathrm{H}_{2} \mathrm{O}-\mathrm{He}$}

We have employed the PES computed by Patkowski et al. [43] for the $\mathrm{H}_{2} \mathrm{O}-\mathrm{He}$ interaction to compute collision integrals for this system. The spherical average was employed in the quantum scattering calculations. The anisotropy of this PES is considerably less than that of the $\mathrm{H}_{2} \mathrm{O}-\mathrm{H}$ PES.

\subsection{Computed Diffusion Coefficients}

Figures 1 and 2 present plots of calculated diffusion coefficients for 8 of the collision systems considered here. Our results are compared conventional calculations using a LJ (12-6) potential, with the parameters taken from the Sandia compilation [44], and several previous calculations using ab initio potentials. Similar comparisons for $\mathrm{OH}-\mathrm{He}$ and $\mathrm{H}_{2} \mathrm{O}-\mathrm{H}$ have been presented previously [25, 27].

We see for most collision pairs that there are significant differences in the computed diffusion coefficients between the quantum scattering and LJ calculations, particularly at high temperatures. In particular, the latter generally are significantly larger than than the former. Previous workers have noted that the repulsive part of the LJ (12-6) potential energy curve is generally steeper and lies at somewhat larger internuclear separations $R$ than for accurate potential energy curves $[8,14,27]$. Thus, the former will lead to larger transport cross sections, and hence smaller diffusion coefficients.

The pronounced exception to this trend is the $\mathrm{H}-\mathrm{H}$ system [see Fig. 1(c)]. The interaction of two $\mathrm{H}\left({ }^{2} S\right)$ atoms involves two potential energy curves, corresponding to the strongly bound $X^{1} \Sigma_{g}^{+}$ground state and the repulsive 

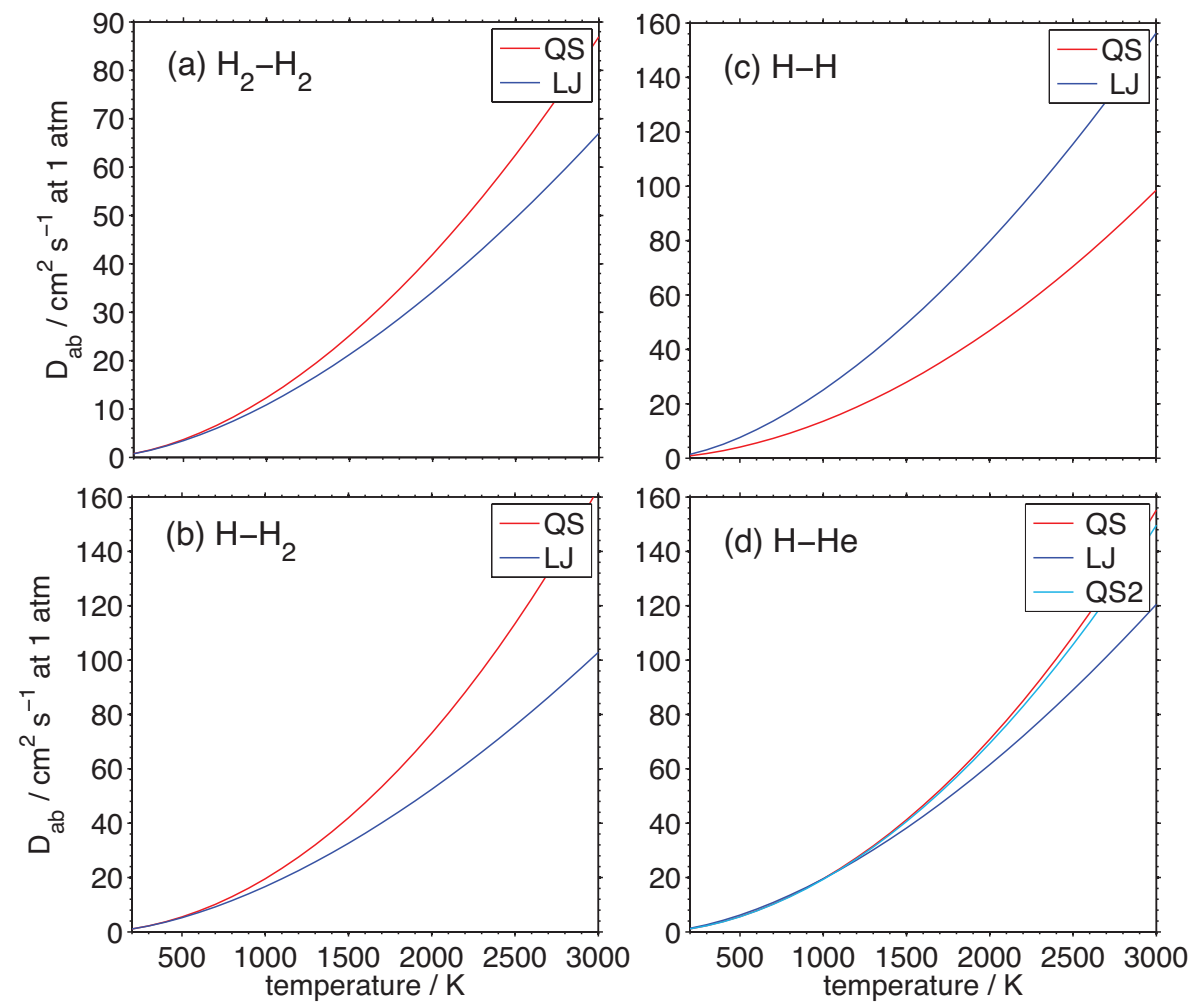

Figure 1: Comparison of binary diffusion coefficients at 1 atm for (a) $\mathrm{H}_{2}-\mathrm{H}_{2}$, (b) $\mathrm{H}-$ $\mathrm{H}_{2}$, (c) H-H, and (d) H-He. QS and LJ denote our quantum scattering calculations and conventional calculations with LJ (12-6) potentials. In panel (d), QS2 denotes the quantum scattering calculation by Middha et al. [22].

triplet $a^{3} \Sigma_{u}^{+}$electronic states. Figure 3 presents the potential energy curves for these states, along with the corresponding LJ (12-6) potential energy curve. We see that the triplet potential energy curve is much more repulsive than the LJ (12-6) potential energy curve. Transport cross sections for this system are spin degeneracy averaged values for the $X^{1} \Sigma_{g}^{+}$and $a^{3} \Sigma_{u}^{+}$state. Hence, the latter is strongly weighted (1:3, respectively), and the $\mathrm{H}-\mathrm{H}$ transport cross section is larger than the LJ value, thus leading to smaller diffusion coefficients for the $\mathrm{H}-\mathrm{H}$ interaction at high temperatures.

For three of the systems for which diffusion coefficients are plotted in Figs. 1 and 2, the results of other quantum scattering calculations are plotted. We see in Fig. 1(c) for $\mathrm{H}-\mathrm{He}$ that there is good agreement of our calculation and 

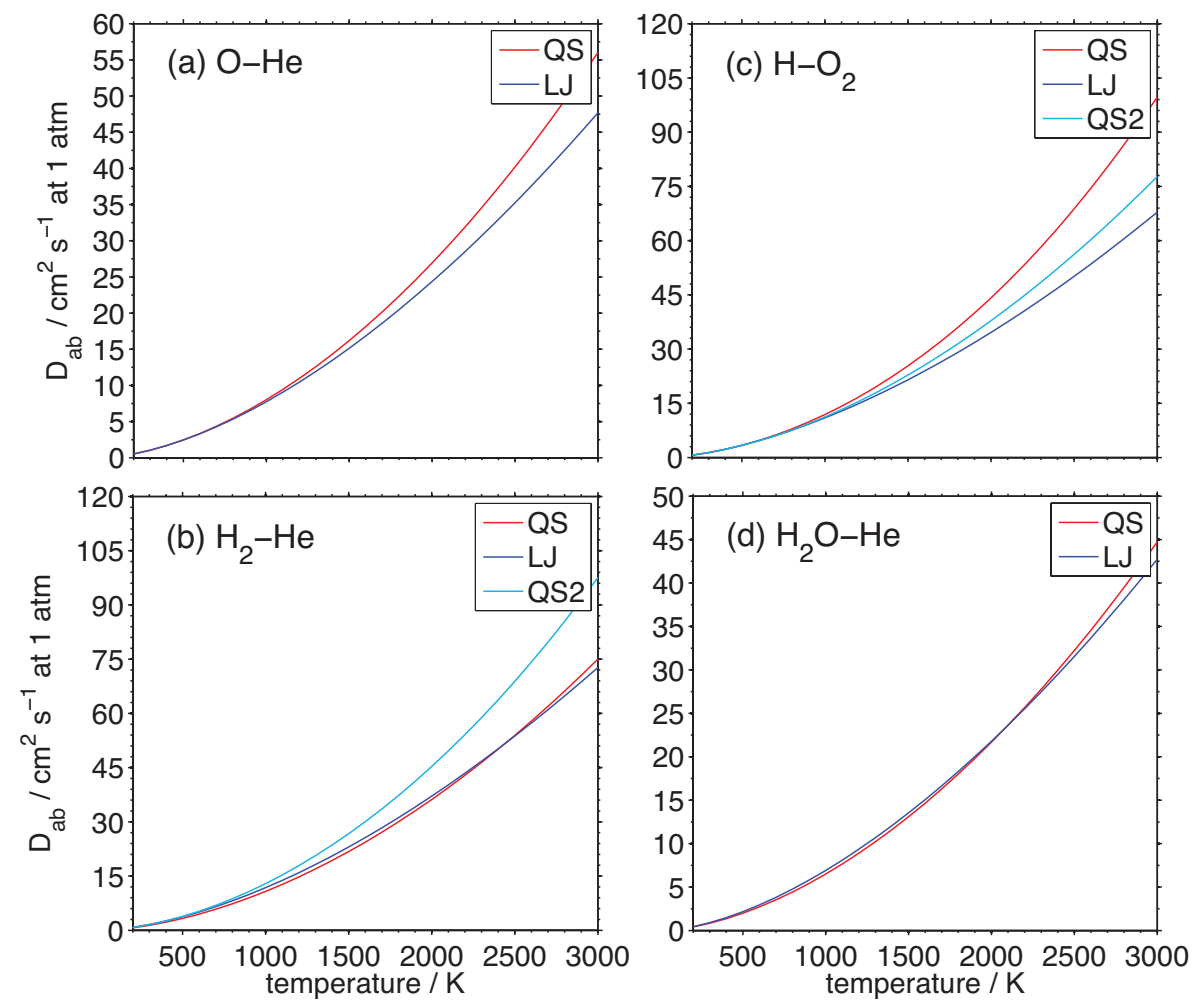

Figure 2: Comparison of binary diffusion coefficients at 1 atm for (a) O-He, (b) $\mathrm{H}_{2}-\mathrm{He}$, (c) $\mathrm{H}_{-} \mathrm{O}_{2}$, and (d) $\mathrm{H}_{2} \mathrm{O}-\mathrm{He}$. QS and LJ denote our quantum scattering calculations and conventional calculations with LJ (12-6) potentials. In panels (b) and (c), QS2 denotes the quantum scattering calculations by Middha et al. [22] and Stallcop et al. [21], respectively.

that of Middha et al. [22]. For $\mathrm{H}_{2}-\mathrm{He}$ [see Fig. 2(b)], the agreement of our calculation and that of Middha et al. [22] is not so good. The origin of the disagreement is not clear.

Our results for $\mathrm{H}-\mathrm{O}_{2}$ differ significantly from the quantum scattering calculations of Stallcop et al. [21] [compare the QS and QS2 curves in Fig. 2(c)]. In their work, they computed the transport properties by considering potential energy curves for the two states $\left[{ }^{2} A^{\prime \prime}\right.$ and ${ }^{4} A^{\prime \prime}$ emanating from $\left.\mathrm{H}\left({ }^{2} S\right)+\mathrm{O}_{2}\left(X^{3} \Sigma_{u}^{-}\right)\right]$only for one orientation angle $\theta$, namely the angle for which $P_{2}(\cos \theta)=0$. By contrast, we carried out full quantum scattering calculations with the 2-dimensional PES's [28]. The anisotropies of the PES's are sufficiently large that the full anisotropic PES's need to be employed in 


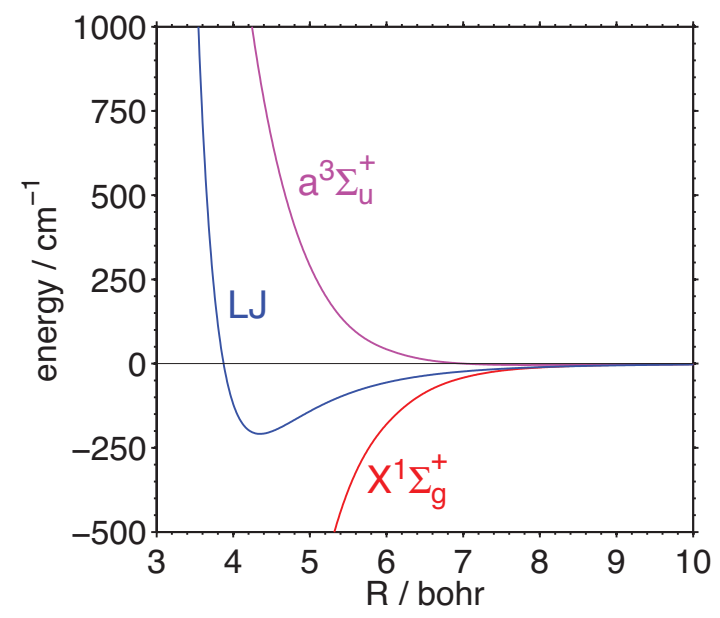

Figure 3: Potential energy curves for the $\mathrm{H}-\mathrm{H}$ interaction.

the calculation of the transport properties [see Fig. 1 of [28] for $(R, \theta)$ contour plots of the PES's].

\section{Combustion Simulations}

The combustion simulations carried out in this work employed the Premix code [45] that is part of the CHEMKIN suite of programs. Calculations were carried out with two sets of transport data. For the first, the binary file of transport property data required by Premix was computed by an unaltered version of Tranfit [44], in which the diffusion coefficients and the collision integral ratios $A^{*}, B^{*}$, and $C^{*}$ were computed with the conventional approach employing parameterized LJ (12-6) potentials. A second set of combustion simulations was performed using a binary file of transport properties computed with a modified version of Tranfit. Here, transport properties were computed from accurate values of the collision integrals for the collision pairs described above and LJ (12-6) transport properties were taken for the other collision pairs. The collision pairs for which accurate transport properties were used include: $\mathrm{H}_{2}-\mathrm{H}_{2}, \mathrm{H}-\mathrm{H}_{2}, \mathrm{H}-\mathrm{H}, \mathrm{H}-\mathrm{O}_{2}, \mathrm{H}-\mathrm{H}_{2} \mathrm{O}, \mathrm{H}-\mathrm{He}, \mathrm{O}-\mathrm{He}, \mathrm{H}_{2}-\mathrm{He}$, 

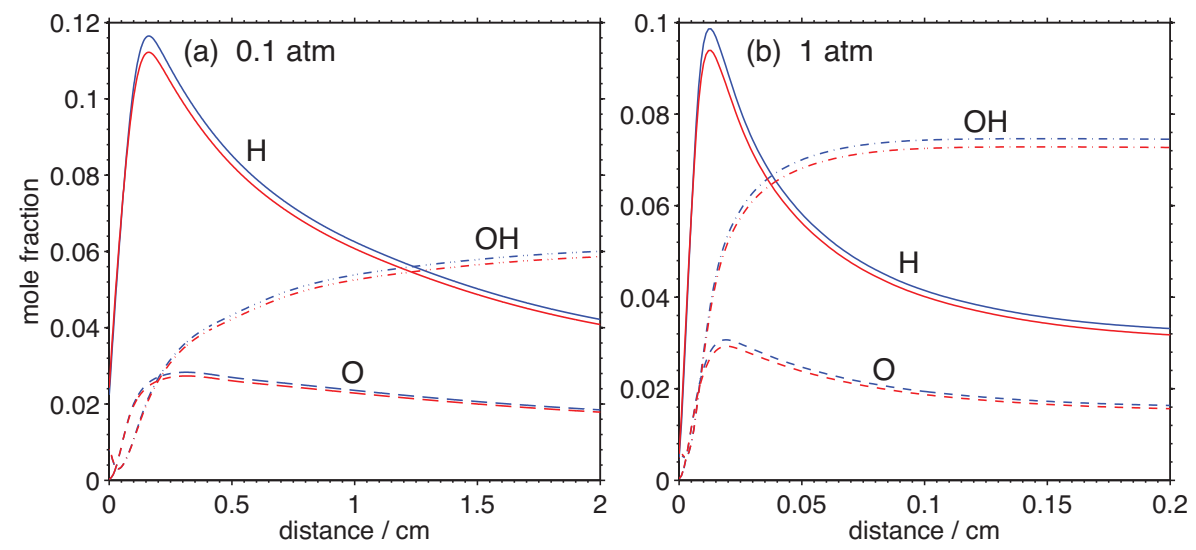

Figure 4: Computed concentration profiles for the $\mathrm{H}$ atom, $\mathrm{O}$ atom, and $\mathrm{OH}$ radical reactive intermediates in a burner stabilized stoichiometric $\mathrm{H}_{2} / \mathrm{O}_{2} / \mathrm{He}$ flame: (a) pressure $1 \mathrm{~atm}$ and flow rate $0.2 \mathrm{~g} \mathrm{~cm}^{2} \mathrm{~s}^{-1}$ and (a) pressure $0.1 \mathrm{~atm}$ and flow rate $0.01 \mathrm{~g} \mathrm{~cm}^{2} \mathrm{~s}^{-1}$. The blue and red curves denotes concentrations computed with LJ and modified transport properties, respectively. The mole fractions of the feed gas were $\mathrm{H}_{2} 0.30, \mathrm{O}_{2}, 0.60, \mathrm{He}$, 0.1 .

\section{$\mathrm{H}_{2} \mathrm{O}-\mathrm{He}$, and $\mathrm{OH}-\mathrm{He}$.}

The $\mathrm{H}_{2} / \mathrm{O}_{2}$ chemistry model employed in this work was taken from the work by Burke et al. [6]. For simulation of a $\mathrm{CH}_{4} / \mathrm{O}_{2}$ flame, the GRI-Mech 3.0 chemistry model [46] was used. In the simulations, the temperature was computed from the computed energy-species equations. The mixturedependent transport properties were calculated using the multicomponent formulation for transport properties [33]. Thermal diffusion was included in the calculations.

In the next section, we present computed laminar flame speeds for premixed, adiabatic, freely propagating $\mathrm{H}_{2} / \mathrm{O}_{2} / \mathrm{He}$ and $\mathrm{CH}_{4} / \mathrm{O}_{2}$ flames.

\section{Results}

Figure 4 presents computed concentration profiles of the $\mathrm{H}$ atom, $\mathrm{O}$ atom, and $\mathrm{OH}$ radical reactive intermediates for a burner stabilized stoichiometric $\mathrm{H}_{2} / \mathrm{O}_{2} / \mathrm{He}$ flame at two pressures. As expected, we see that the region of high concentration of these reactive intermediates is larger at the lower pressure. The $\mathrm{H}$ atom mole fraction reaches a peak of $\sim 0.1$ at both pressures, with slightly lower mole fractions for $\mathrm{O}$ and $\mathrm{OH}$. Of particular interest for this 


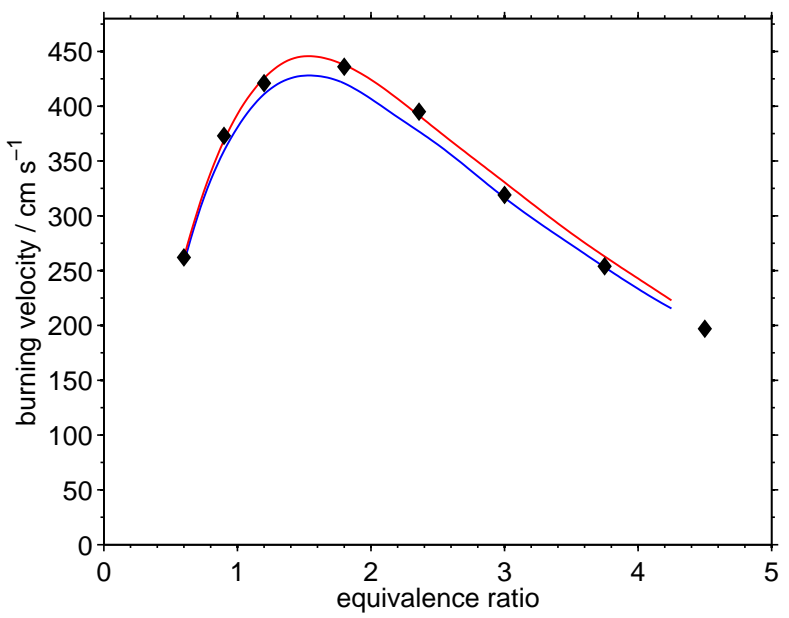

Figure 5: Laminar flame speed at $1 \mathrm{~atm}$ for $\mathrm{H}_{2} / \mathrm{O}_{2}$ diluted in $\mathrm{He}$ with a $\mathrm{O}_{2} / \mathrm{He}$ dilution ratio of 3.76. The blue and red curves denote calculations with LJ and modified transport properties, respectively. The black diamonds are experimental data from Kwon and Faeth $[47]$.

study are the differences in the computed concentrations when the LJ or modified transport properties are employed in the combustion simulations. The concentrations computed with the latter set of transport properties are slightly less than when LJ transport properties are used. We also see that the differences in the concentrations between the two sets of calculations are similar at the two pressures.

Figure 5 presents calculated flame speeds for a $\mathrm{H}_{2} / \mathrm{O}_{2} / \mathrm{He}$ flame at $1 \mathrm{~atm}$, computed with LJ transport properties and our set of transport properties modified to include accurate values for selected collision pairs. The flame speeds were computed as a function of the equivalence ratio and are compared with experimental data from Kwon and Faeth [47].

Both simulations are in good agreement with the experimental measurements. We see that there is a modest difference in the flame speeds computed with the two sets of transport properties. The fractional difference in flame speeds is slightly larger than the differences in computed radical concentrations displayed in Fig. 4. Flame speeds computed with the modified set of transport properties are slightly larger than those computed with LJ transport properties. The difference in the computed flame speeds is slightly greater at the higher equivalence ratios. We also find that the difference is 


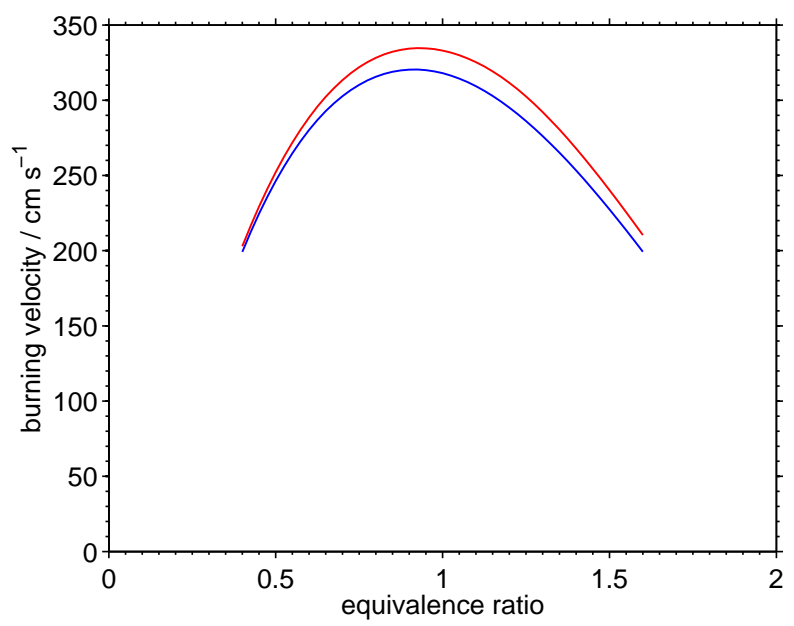

Figure 6: Laminar flame speed at 1 atm for $\mathrm{CH}_{4} / \mathrm{O}_{2}$ vs. the equivalence ratio. The blue and red curves denote calculations with LJ and modified transport properties, respectively.

comparable to the difference in flame speeds computed with different recent chemistry models $[6,48]$ (see, for example, computed flame speeds in Fig. 20 of Burke et al. [6]).

We also investigated the calculation of laminar flame speeds using accurate diffusion coefficients for our selected collision pairs, but with LJ values of the collision integral ratios $A^{*}, B^{*}$, and $C^{*}$ for all collision pairs. In this case, the maximum flame speed, namely $540 \mathrm{~cm} \mathrm{~s}^{-1}$ at an equivalence ratio of 1.6, was found to be much higher than for the curves plotted in Fig. 5. This value seems suspect, since we are not computing all the transport properties for each of the collision pairs from the same potential.

We have also investigated the use of accurate transport properties for our subset of collision pairs in $\mathrm{CH}_{4} / \mathrm{O}_{2}$ premixed flames. In this case, the chemistry model [46] includes many more species than for a $\mathrm{H}_{2} / \mathrm{O}_{2}$ flame. Figure 6 presents computed laminar flame speeds for a range of equivalence ratios for a $\mathrm{CH}_{4} / \mathrm{O}_{2}$ flame at $1 \mathrm{~atm}$, computed with LJ transport properties and our set of transport properties modified to include accurate values for the selected collision pairs.

Again, we see a difference in the flame speeds computed with the two sets of transport properties, with higher flame speeds found for the modified set of transport properties. The differences are also larger at higher equivalence 
ratios. Despite the greater number of chemical species in $\mathrm{CH}_{4}$ vs. $\mathrm{H}_{2}$ combustion, the differences in the computed flame speeds are, in fact, slightly greater for the former. The flame kinetics are expected to be most sensitive to the transport properties of lighter species, especially the $\mathrm{H}$ atom. In our modified set of transport properties, we have included accurate values for a number of collision pairs involving the $\mathrm{H}$ atom.

To our knowledge, experimental laminar flame speeds have not been reported for $\mathrm{CH}_{4} / \mathrm{O}_{2}$ flames, although there have been numerous reports of measured flame speeds for $\mathrm{CH}_{4}$ /air flames, reviewed by Lowry et al. [49]. We defer to a future publication comparison of measured flame speeds of $\mathrm{CH}_{4} /$ air flames with our combustion simulations with accurate transport properties to when we have computed these for more collision pairs, particularly those involving carbon-containing species.

\section{Discussion}

In this paper, we have investigated the effect of employing accurate transport properties for a subset of collision pairs, mainly for pairs involving the light $\mathrm{H}$ atom, in the calculation of laminar flame speeds for premixed, freely propagating flames. For the two flames investigated, we find a modest increase in computed flame speeds when the accurate transport properties are incorporated into the transport database. This increase is, in fact, comparable to differences in computed flame speeds when different chemistry models are employed, at least for $\mathrm{H}_{2} / \mathrm{O}_{2} / \mathrm{He}$ flames. This is consistent with previous sensitivity analyses in which it was concluded that inaccuracies in certain transport properties are comparable to uncertainties in reaction rate constants in flame simulations $[7,8,9,10]$.

It is obviously impractical to compute accurate transport properties for a significant fraction of the large number of collision pairs in the air combustion of methane and larger hydrocarbons. However, since transport of the light $\mathrm{H}$ atom and $\mathrm{H}_{2}$ will be more important than transport of other, heavier species, it is appropriate to concentrate on collision systems involving this atom. Indeed, for the most important collision pair in air oxidation, namely $\mathrm{H}-\mathrm{N}_{2}$, accurate transport properties have already been computed [13, 21, 24]. Other collision pairs for which accurate transport properties should be computed include $\mathrm{H}-\mathrm{CO}$ and $\mathrm{H}-\mathrm{CO}_{2}$. The author is presently engaged in calculating accurate transport properties for these and other collision pairs. 
We make one further point. In the multicomponent model for transport properties [33], both the diffusion coefficient and the collision integral ratios $A^{*}, B^{*}$, and $C^{*}$ are required to treat transport in mixtures. It would thus not be advisable to employ accurately computed diffusion coefficients without also doing the same for the collision integral ratios.

\section{Acknowledgements}

The author is very grateful to Raghu Sivaramakrishnan for providing the publicly available Fortran source codes of the CHEMKIN program suite. In addition, he kindly answered a number of questions about combustion simulations. The author also acknowledges helpful correspondence with Hai Wang. This material is based upon work supported by the U.S. Department

of Energy, Office of Science, Office of Basic Energy Sciences, under Grant No. DESC0002323.

\section{Appendix A. Supplementary Material}

Supplementary data associated with this article can be found, in the online version, at [to be filled in by the journal].

\section{References}

[1] M. D. Smooke, The computation of laminar flames, Proc. Comb. Inst. 34 (2013) 65-98.

[2] C. K. Westbrook, F. L. Dryer, Chemical kinetic modeling of hydrocarbon combustion, Prog. Energy Comb. Sci. 10 (1984) 1-57.

[3] J. A. Miller, C. T. Bowman, Mechanism and modeling of nitrogen chemistry in combustion, Prog. Energy Combust. Sci. 15 (1989) 287-3398.

[4] J. A. Miller, M. J. Pilling, J. Troe, Chemical-kinetics and combustion modeling, Annu. Rev. Phys. Chem. 41 (1990) 345-387.

[5] Z. Hong, D. F. Davidson, R. K. Hanson, An improved $\mathrm{H}_{2} / \mathrm{O}_{2}$ mechanism based on recent shock tube/laser absorption measurements, Comb. Flame 158 (2011) 633-644. 
[6] M. P. Burke, M. Chaos, J. Y., F. L. Dryer, S. J. Klippenstein, Comprehensive $\mathrm{H}_{2} / \mathrm{O}_{2}$ kinetic model for high-pressure combustion, Int. J. Chem. Kinet. 44 (2012) 444-474.

[7] N. J. Brown, K. L. Revzan, Comparative sensitivity analysis of transport properties and reaction rate coefficients, Int. J. Chem. Kinet. 37 (2005) $538-553$.

[8] Y. Dong, A. T. Holley, M. G. Andac, F. N. Egolfopoulos, S. G. Davis, P. Middha, H. Wang, Extinction of premixed $\mathrm{H}_{2}$ /air flames: Chemical kinetics and molecular diffusion effects, Comb. Flame 142 (2005) 374387.

[9] G. Esposito, H. K. Chelliah, Effect of binary diffusion and chemical kinetic parameter uncertainties in simulations of premixed and nonpremixed laminar hydrogen flames, Comb. Flame 159 (2012) 3522-3529.

[10] G. Esposito, B. G. Sarnacki, H. K. Chelliah, Uncertainty propagation of chemical kinetics parameters and binary diffusion coefficients in predicting extinction limits of hydrogen/oxygen/nitrogen non-premixed flames, Comb. Theory Modeling 16 (2012) 1029-1052.

[11] N. J. Brown, L. A. J. Bastien, P. N. Price, Transport properties for combustion modeling, Prog. Energy Combust. Sci. 37 (2011) 565-582.

[12] A. W. Jasper, J. A. Miller, Lennard-jones parameters for combustion and chemical kinetics modeling from full-dimensional intermolecular potentials, Combust. Flame 161 (2014) 101-110.

[13] E. Jasper, A. W. Kamarchik, J. A. Miller, S. J. Klippenstein, Firstprinciples diffusion coefficients for $\mathrm{H}, \mathrm{H}_{2}$, and four normal alkanes $+\mathrm{N}_{2}$, J. Chem. Phys. 141 (2014) No. 124313.

[14] P. Paul, J. Warnatz, A re-evlauation of the means used to calculate transport properties of reacting flows, Proc. Combust. Inst. 27 (1998) 495-504.

[15] J. R. Stallcop, H. Partridge, S. P. Walsh, E. Levin, H- $\mathrm{N}_{2}$ interaction energies, transport cross sections, and collision integrals, J. Chem. Phys. 97 (1992) 3431-3436. 
[16] J. R. Stallcop, H. Partridge, E. Levin, $\mathrm{H}-\mathrm{H}_{2}$ collision integrals and transport coefficients, Chem. Phys. Lett. 254 (1996) 25-31.

[17] J. R. Stallcop, H. Partridge, E. Levin, Ab initio potential-energy surfaces and electron-spin-exchange cross sections for $\mathrm{H}-\mathrm{O}_{2}$ interactions, Phys. Rev. A 53 (1996) 766-771.

[18] J. R. Stallcop, E. Levin, H. Partridge, Transport properties of hydrogen, J. Thermophys. Heat Trans. 12 (1998) 514-519.

[19] J. R. Stallcop, H. Partridge, E. Levin, Effective potential energies and transport cross sections for interactions of hydrogen and nitrogen, Phys. Rev. A 62 (2000) No. 062709.

[20] J. R. Stallcop, H. Partridge, A. Pradhan, E. Levin, Potential energies and collision integrals for interactions of carbon and nitrogen atoms, J. Thermophys. Heat Trans. 14 (2000) 480-488.

[21] J. R. Stallcop, H. Partridge, E. Levin, Effective potential energies and transport cross sections for atom-molecule interactions of nitrogen and oxygen, Phys. Rev. A 64 (2001) No. 042722.

[22] P. Middha, B. H. Yang, H. Wang, A first-principle calculation of the binary diffusion coefficients pertinent to kinetic modeling of hydrogen/oxygen/helium flames, Proc. Combust. Inst. 29 (2002) 1361-1369.

[23] P. Middha, H. Wang, First-principle calculation for the hightemperature diffusion coefficients of small pairs: The $\mathrm{H}-\mathrm{Ar}$ case, Combust. Theory Modeling 9 (2005) 353-363.

[24] A. S. Dickinson, A. Ern, V. Vesovic, Transport properties of $\mathrm{H}-\mathrm{N}_{2}$ mixtures, Mol. Phys. 103 (2005) 1985-1904.

[25] P. J. Dagdigian, M. H. Alexander, Exact quantum scattering calculation of transport properties for free radicals: $\mathrm{OH}\left(X^{2} \pi\right)$-helium, J. Chem. Phys. 137 (2012) No. 094306.

[26] P. J. Dagdigian, M. H. Alexander, Exact quantum scattering calculation of transport properties: $\mathrm{CH}_{2}\left(\tilde{X}^{3} B_{1}, \tilde{a}^{1} A_{1}\right)$-helium, J. Chem. Phys. 138 (2013) No. 164305. 
[27] P. J. Dagdigian, M. H. Alexander, Exact quantum scattering calculation of transport properties for the $\mathrm{H}_{2} \mathrm{O}-\mathrm{H}$ system, J. Chem. Phys. 139 (2013) No. 194309.

[28] P. J. Dagdigian, M. H. Alexander, Transport properties for systems with deep potential wells: $\mathrm{H}+\mathrm{O}_{2}$, J. Phys. Chem. A (2014) (in press).

[29] J. O. Hirschfelder, C. F. Curtiss, R. B. Bird, Molecular Theory of Gases and Liquids, Wiley, New York, 1954.

[30] L. Monchick, K. S. Yun, E. A. Mason, Formal kinetic theory of transport phenomena in polyatomic gas mixtures, J. Chem. Phys. 39 (1963) 654669.

[31] G. C. Maitland, M. Mustafa, W. A. Wakeham, F. R. W. McCourt, An essentially exact evaluation of transport cross-sections for a model of the helium-nitrogen interaction, Mol. Phys. 61 (2) (1987) 359-387.

[32] F. R. W. McCourt, J. J. M. Beenakker, W. E. Köhler, I. Kuščer, Nonequilibrium Phenomena in Polyatomic Gases, Clarendon Press, Oxford, 1990.

[33] G. Dixon-Lewis, Flame structure and flame reaction kinetics. 2. transport phenomena in multicomponent systems, Proc. R. Soc. London A 307 (1968) 111-135.

[34] MOLPRO, version 2010.1, a package of ab initio programs by H.J. Werner, P. J. Knowles, R. Lindh, F. R. Manby, M. Schütz, and others, see http://www.molpro.net.

[35] C. Hampel, K. A. Petersen, H.-J. Werner, A comparison of the efficiency and accuracy of the quadratic configuration-interaction (QCISD), coupled cluster (CCSD), and Bruekner coupled cluster (BCCD) methods, Chem. Phys. Lett. 190 (1992) 1-12.

[36] M. Deegan, P. J. Knowles, Perturbative corrections to account for triple excitations in closed and open-shell coupled-cluster theories, Chem. Phys. Lett. 227 (1994) 321-326.

[37] R. A. Kendall, T. H. Dunning, Jr., R. J. Harrison, Electron-affinities of the first-row atoms revisited. systematic basis sets and wave functions, J. Chem. Phys. 96 (1992) 6796-6806. 
[38] S. F. Boys, F. Bernardi, Calculation of small molecular interactions by differences of separate total energies. some procedures with reduced errors, Mol. Phys. 19 (1970) 553-566.

[39] R. Krems, A. A. Buchachenko, M. M. Szcześniak, J. Kłos, G. Chałasiński, Dynamics of $\mathrm{O}\left({ }^{3} \mathrm{P}_{j}\right)+\mathrm{Rg}$ collisions on ab initio and scattering potentials, J. Chem. Phys. 116 (2002) 1457-1467.

[40] M. H. Alexander, T. Orlikowski, J. E. Straub, Theoretical study of intramultiplet transitions in collisions of atoms in ${ }^{3} \mathrm{P}$ electronic states with structureless targets: $\mathrm{Ca}\left({ }^{3} \mathrm{P}\right)+\mathrm{He}$, Phys. Rev. A 26 (1983) 73-82.

[41] B. W. Bakr, D. G. A. Smith, K. Patkowski, Highly accurate potential energy surface for the $\mathrm{HeH}_{2}$ dimer, J. Chem. Phys. 139 (2013) No. 144305 .

[42] A. M. Arthurs, A. Dalgarno, The theory of scattering by a rigid rotator, Proc. R. Soc. London Ser. A 256 (1287) (1960) 540-551.

[43] K. Patkowski, T. Korona, R. Moszynski, B. Jeziorski, K. Szalewicz, Ab initio potential energy surface and second virial coefficient for $\mathrm{He}-\mathrm{H}_{2} \mathrm{O}$ complex, J. Mol. Struct. (Theochem) 591 (2002) 231-243.

[44] R. J. Kee, G. Dixon-Lewis, J. Warnatz, M. E. Coltrin, and J. A. Miller, A Fortran Computer Code Package for the Evaluation of Gas-Phase Viscosities, Conductivities, and Diffusion Coefficients, Report SAND868246, Sandia National Laboratories, 1986.

[45] R. J. Kee, J. F. Grcar, M. S. Smooke, and J. A. Miller, PREMIX: A Fortran Program for Modeling Steady Laminar One-Dimensional Premixed Flames, Report SAND85-8240, Sandia National Laboratories, 1985.

[46] GRI-Mech 3.0, The Gas Research Institute, http:// www.me.berkeley.edu/gri_mech/.

[47] O. D. Kwon, G. M. Faeth, Flame/stretch interactions of premixed hydrogen-fueled flames: Measurements and predictions, Comb. Flame 124 (2001) 590-610.

[48] J. Li, Z. Zhao, A. Kazakov, F. L. Dryer, An updated comprehensive kinetic model of hydrogen combustion, Int. J. Chem. Kinet. 36 (2004) $566-575$. 
[49] W. Lowry, J. de Vries, M. Krejci, E. Petersen, Z. Serinyel, W. Metcalfe, H. Curran, G. Bourque, Laminar flame speed measurements and modeling of pure alkanes and alkane blends at elevated pressures, J. Eng. Gas Turbines Power 133 (2011) No. 091501. 